\title{
Very Fast Similarity Queries on Semi-Structured Data from the Web
}

\author{
Bhavana Dalvi \\ School of Computer Science \\ Carnegie Mellon University \\ bbd@cs.cmu.edu
}

\author{
William W. Cohen \\ School of Computer Science \\ Carnegie Mellon University \\ wcohen@cs.cmu.edu
}

\begin{abstract}
In this paper, we propose a single low-dimensional representation for entities found in different datasets on the web. Our proposed PIC-D embeddings can represent large D-partite graphs using small number of dimensions enabling fast similarity queries. Our experiments show that this representation can be constructed in small amount of time (linear in number of dimensions). We demonstrate how it can be used for variety of similarity queries like set expansion, automatic set instance acquisition, and column classification. Our approach results in comparable precision with respect to task specific baselines and up to two orders of magnitude improvement in terms of query response time.
\end{abstract}

\section{Introduction}

On the web, many entities are mentioned many times, in many contexts. In particular, many entities appear frequently in hyponym patterns or "Hearst patterns" [8] (e.g., "cities such as New York", or more abstractly, "concept such as entity") and also in semi-structured web pages that can be easily parsed into tables and lists. In past work, hyponym and table data about entities has been used to address a number of distinct tasks, such as fact extraction (e.g., [5]), semi-supervised learning (e.g., [14,3]), set expansion (e.g., [19]), determining the type of columns in tables (e.g., [9]), and automatic set instance acquisition (e.g., [18]).

However, existing methods to solve these tasks make quite different use of the underlying data: for instance, SEAL [19] performs set expansion by performing web queries and recognizing "wrappers" for semi-structured pages and merging the results on-the-fly, while WebTables [2] uses a precomputed collection of tables with header information to perform schema auto-completion. Label propagation methods like MAD [14] can address multiple such tasks using the same graph based representation, but results in huge query response times. Having diverse methods for processing web data, with diverse storage and access requirements, can lead to unnecessary inefficiencies and complexities.

Let us consider a scenario where there is a large set of entities $x_{1}, \ldots, x_{n}$, small number $m$ of them are labeled $\left(x_{1}, y_{1}\right), \ldots,\left(x_{m}, y_{m}\right)$. We have $D$ different datasets which has co-occurrence information about $x_{i}$ 's. Some examples of such datasets are co-occurrence of entities in HTML table columns on the Web pages, occurrences of entities in the free text around Hearst patterns [8], occurrences of entities as Subject-Verb-Object triples etc. Our focus is on representing this high-dimensional data about entities in a low dimensional representation so that, many similarity queries can be efficiently executed against this presentation. Consider following three tasks:

1. Set Expansion (SE) [19]: Here the query is a set of seed entities $\left\{x_{1}, \ldots, x_{s}\right\}$ of a single class $y$, and the task is to return a larger set of entities from the same class (e.g., given a set of three seed baseball teams, find more teams from the same league.)

2. Concept name expansion, or "automatic set instance acquisition" (ASIA) [18]: Here the query is a single class name (e.g., "baseball teams"). The task is to return a set of instances of that concept.

3. Column Classification (COL-CLASS) [9]: Here again we have a set of seed examples and unlabeled examples. The task is to learn a classifier that can predict the class label $y_{c}$ for an unlabeled table column $c$ where $c$ is a set of examples $c=\left\{x_{1}, \ldots, x_{c}\right\}$.

Note that $x_{i}$ 's mentioned here are entities, and the above mentioned tasks can be considered as similarity queries because they rely on putting similar entities closer to each other in the data space. Our hypothesis is that If we can learn a low dimensional data representation in which entities belonging to the same concept are together, we can solve the above mentioned tasks efficiently operating in this low dimensional space.

Contributions of this paper are as follows : (1) We propose a low-dimensional representation (henceforth referred to as the PIC-D representation) and handle the distinct tasks listed above as similarity queries to be executed against this representation. (2) Further we demonstrate that the 
PIC-D representation can be created very quickly, in time linearly proportional to the total number of dimensions in the dataset. Once this pre-processing is done, queries run much faster in the lower-dimensional space. (3) We compare our proposed approach with baseline approaches like similarity queries over the original high-dimensional space and state of the art label propagation techniques [14].

We are interested in representing the data extracted from the web, hence we consider three representations that can be constructed efficiently for very large datasets. One such scheme is to represent each entity as a sparse vector of all its occurrences in all datasets. Another potential scheme is a graph, in which entities along with all features are nodes and edges represent co-occurrence of entities with features. Finally, we consider our proposed PIC-D representation for this graph, which internally uses an efficient network clustering method called Power Iteration Clustering [11].

To demonstrate our representation, we use hyponym and semi-structured occurrence data from the web, and represent it as a tri-partite graph. Our experiments show that this representation is generally preferable to other methods, especially with respect to performance at query time (12x to $240 \mathrm{x}$ faster than the sparse representation and $65 \mathrm{x}$ to $300 \mathrm{x}$ faster when compared to state of the art label propagation technique [14]) at the expense of modest pre-processing time (around $365 \mathrm{msec}$. for a graph with half a million edges).

Below, Section 2 describes our low-dimensional representation named PIC-D representation. Then Section 3 discusses how can we make sure that the PIC-D is a reasonable representation of the underlying data. Section 4 demonstrates the use of this representation for the previously mentioned similarity queries. Experimental results on various semi-structured datasets are described in Section 5. Section 6 discusses previous work in this research area, and finally we present our conclusions in Section 7 .

\section{The PIC-D Representation}

We propose a low-dimensional representation for entities based on the embedding internally used by the PIC algorithm [11]. Briefly, PIC assigns each node in a graph an initial random numeric value, and then performs an iterative update which brings together the values assigned to nearby nodes, thus producing a one-dimensional embedding of a graph.

Algorithm 1 describes the procedure of creating the PIC-D representation for a $(D+1)$-partite graph containing entity occurrences in $D$ different datasets. Figure 1 shows the schematic diagram of intermediate matrices while creating this embedding. Specifically, we start with $m$ random vectors to generate $m$-dimensional PIC embedding for each bi-partite graph corresponding to each dataset. Since we have $D$ such bipartite graphs, we create embeddings for each of them separately and final PIC-D embedding is the concatenation of these individual embeddings. (See Section
1: function Create_PIC-D_Embedding $\left(m, X, X_{1}, X_{2}, \ldots, X_{D}\right)$ : $X_{\mathrm{PIC}-\mathrm{D}}$

2: Input: $m$ : Number of PIC dimensions per dataset $X$ : Set of all entities,

$X_{1}$ : Co-occurrence of $X$ in dataset $_{1}$,

$X_{2}$ : Co-occurrence of $X$ in dataset $_{2}$,

....

$X_{D}$ : Co-occurrence of $X$ in dataset $_{D}$,

3: Output: $X_{\text {PIC-D }}:(|X|, D * m)$ dim. embedding of $X$.

4: $X_{\text {PIC-D }}=\phi$

5: $t=$ a small positive integer

6: for $i=1$ : $D$ do

7: for $\mathrm{j}=1: \mathrm{m}$ do

8: $\quad V_{0}=$ randomly initialized vector of size $|X| * 1$

9: $\quad V_{t}=$ PIC_Embedding $\left(X_{i}, V_{0}, t\right)$

10: $\quad$ Append $V_{t}$ as a new column $X_{\text {PIC-D }}$

11: end for

12: end for

13: end function

Algorithm 1: Create PIC-D embedding

5.2 gives some insights on how to choose a right value of $m$ ).

We have experimented with a version of this algorithm in which we create PIC embeddings of the data by concatenating the dimensions first instead of computing separate embeddings and later concatenating them. We observed that the version showed in Algorithm 1 performs as good as or better than its variant. Further, PIC is proved to be equivalent to computing diffusion maps followed by random projection of points in a one dimensional space ([10] Section 3.6). Instead PIC-D representation can be considered as random projection in $m * D$ dimensional space.

We use Algorithm 1 to produce a low-dimensional embedding of a tripartite graph, in particular the data graph of Figure 2(a). This tri-partite graph is populated using entitytableColumn co-occurrence dataset and Hyponym Concept dataset published by Dalvi et al.[5]. Each edge derived from the entity-tableColumn dataset links an entity name with an identifier for a table column in which the entity name appeared. Each edge derived from the Hyponym Concept Dataset links an entity $\mathrm{X}$ and a concept $\mathrm{Y}$ with which it appeared in the context of a Hearst pattern (weighted by frequency in a large web corpus). We combine these edges to form a tripartite graph, as shown in Figure 2 (a). Occurrences of entities with hyponym (or "such as") concepts form a bipartite graph on the left, and occurrences of entities in various table-columns form the bipartite graph on the right. Our hypothesis is that entities co-occurring in multiple table columns and/or with similar suchas concepts might belong to the same class label.

Here we have two bipartite graphs, entity-tableColumn and entity-suchasConcept. We create bipartite PIC embeddings for each of these in turn (retaining only the part of the embedding relevant to the entities). The embedding for enti- 


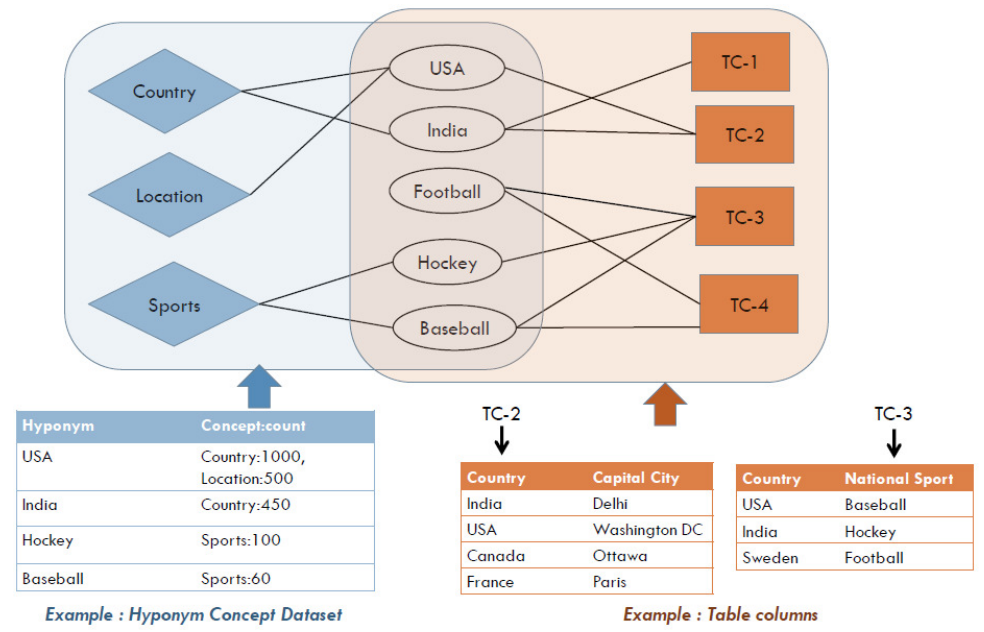

(a) Example Tri-partite Graph

\begin{tabular}{|l|l|l|l|l|}
\hline Entity & $\mathbf{X}_{1}$ & $\mathbf{X}_{2}$ & $Y_{1}$ & $Y_{2}$ \\
\hline USA & 0.23 & 0.76 & 0.43 & 0.66 \\
\hline India & 0.21 & 0.79 & 0.41 & 0.69 \\
\hline Football & 0.36 & 0.80 & 0.66 & 0.35 \\
\hline Hockey & 0.35 & 0.82 & 0.16 & 0.92 \\
\hline Baseball & 0.34 & 0.79 & 0.14 & 0.89 \\
\hline
\end{tabular}

(b) Example PIC-D representation $(m=2)$

Figure 2: Example PIC-D embeddings

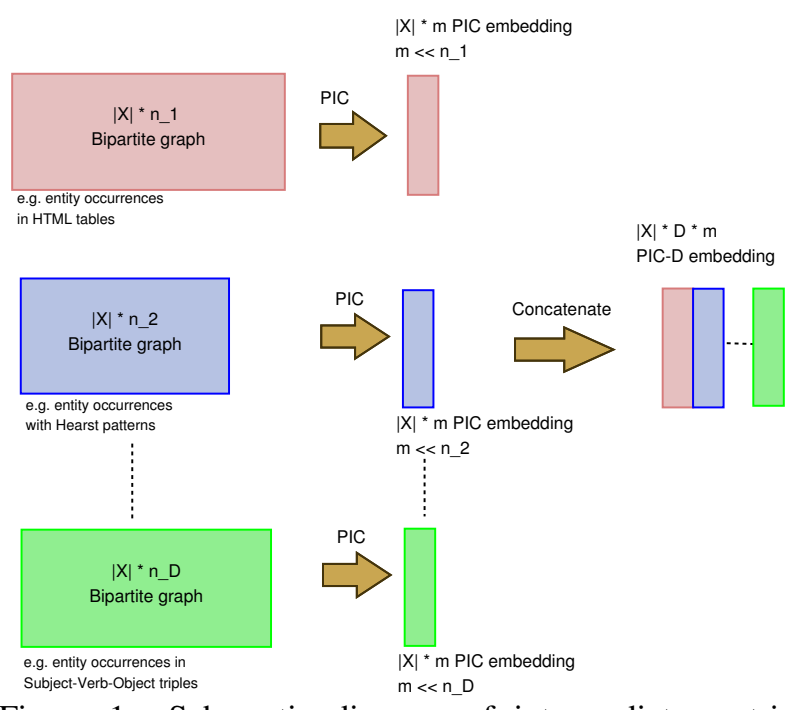

Figure 1: Schematic diagram of intermediate matrices, where $|X|$ : number of entities, $n_{i}$ : number of dimensions in Dataset $_{i}, \mathrm{n}$ : total number of dimensions $\left(n=\sum_{i} n_{i}\right)$, and $\mathrm{m}$ : number of PIC dimensions per bipartite graph.

ties is then the concatenation of these separate embeddings.

Our hypothesis is that these embeddings will cluster similar entities together. Figure 2 (b) shows an example PIC-D embedding of the tri-partite graph with $d=2$ and $m=2$. Columns X1 and X2 denote the PIC embeddings corresponding to entity-tableColumn bipartite graph, while $\mathrm{Y} 1$ and $\mathrm{Y} 2$ represent the embeddings corresponding to entity-suchas bipartite graph. In the next section, we will discuss how to evaluate whether the PIC-D embeddings are reasonable.

\section{Is the PIC-D representation reasonable?}

We would like the PIC-D representation to capture the properties of the underlying dataset, so that similar entities (belonging to the same concept) are put closer to each other in the new low-dimensional space. In this section we will discuss how to tune the parameters of Algorithm 1 to generate a reasonable PIC-D representation.

Parameter Tuning There are two parameters in Algorithm 1 (1) $t$ : number of iterations of PIC algorithm and (2) $m$ : number of dimensions of the PIC-D embedding. We set $t$ to a small number (say 5) to generate independent embeddings starting with different random initialization vectors. We then note that the effectiveness of the PIC-D representation depends on parameter $m$.

We use semi-supervised learning task to measure the effectiveness of the PIC-D representation. Here the query is a small set of labeled "seed" examples $\left(x_{1}, y_{1}\right), \ldots,\left(x_{m}, y_{m}\right)$ and many unlabeled instances $x_{m+1}, \ldots, x_{n}$. The task is to label the unlabeled instances accurately with labels $y$ (i.e., we assume a transductive setting [14].)

To use the PIC-D representation for this task, we simply learn a linear classifier in the embedded space. In Section 5.2, we experiment with various datasets to find the relation between ideal value of $m$ for a given dataset with $n$ number of dimensions. We also note that SSL task is much more effective (precision on test data) with the use of PIC-D representation than applying graph-based iterative semi-supervised learning methods [14]. 


\section{Similarity Queries on PIC-D}

In this section we will see how the PIC-D representation for entities can be used for different tasks. In general, our algorithms exploit the property that semantically similar entities are nearby (with respect to Euclidean distance) in the PIC-D space. In the experimental section, we will also discuss baseline approaches to solving each of these tasks using the other representations.

4.1 Set Expansion Set expansion refers to the problem of expanding a set of "seed" entities into a larger set of entities of the same type. Algorithm 2 formally describes how to perform set expansion with the PIC-D representation. Given a set of query entities, the algorithm computes their centroid in PIC-D space. $k$ nearest neighbors are then found by measuring distances from the centroid using a KD-tree. At the end we apply Otsu's threshold the ranked list of entities to get optimal set expansion for a given set of seed entities.

In some prior set expansion papers, the result of set expansion was evaluated as a ranked list [17]; however, here we apply Otsu's thresholding algorithm [13] to select the correct number of results for each query from a large ranked list, following $\mathrm{He}$ and Xin [7]. In our experiments we threshold an ranked initial list of $k=200$ entities. Again, this approach is quite efficient at query time; prior approaches such as SEAL [19] ranked nodes using random-walk-withrestart methods within a graph it built on-the-fly at set expansion time using queries to the web.

1: function Expand_Set $\left(Q, X_{P I C}\right): Q^{\prime}$

2: Input: $Q$ : seed entities for set expansion,

$X_{P I C}$ : low dimensional PIC-D embedding of $E$

3: Output: $Q^{\prime}$ : Expanded entity set

4: $x_{Q}=X_{P I C}($ row,$:)$, row $\in Q$

5: $x_{\text {centroid }}=\operatorname{centroid}\left(x_{Q}\right)$

6: $k=$ a large positive number

7: $\left[Q_{k}\right.$, Score $\left._{k}\right]=$ Find-K-NearestNbr $\left(x_{\text {centroid }}, X_{P I C}, k\right)$

8: $Q^{\prime}=$ Apply-Otsu-Threshold $\left(Q_{k}\right.$, Score $\left._{k}\right)$

9: end function

Algorithm 2: Set Expansion with K-NN on PIC-D

4.2 Automatic Set Instance Acquisition (ASIA) This task takes as input the name of a semantic class (e.g., "countries") and automatically outputs its instances (e.g., "USA", "India", "China" etc.). As described in Algorithm 3, we look up instances of the given class in the hyponym dataset, and then perform set expansion on these a process analogous to that used in a prior work [18]. Here, however, we again use Algorithm 2 for set expansion in the PIC space created using only entity-tableColumn bipartite graph. The entity-suchasConcept data is used only to find seeds for a particular class $Y$. Again this method requires minimal resources at query time.
1: function Set_Instance_Acquisition $\left(y_{s}, X_{P I C}\right): X_{y_{s}}$

2: Input: $y_{s}$ : class name (one of suchas concepts),

$X_{P I C}$ : low dimensional PIC-D embedding of $E$

3: Output: $X_{y_{s}}$ : Entity set belonging to concept $y_{s}$

4: numSeeds = a small positive number

5: seeds $=$ top numSeeds entities that occur with $y_{s}$ in Hyponym Concept Dataset

6: $X_{y_{s}}=$ Expand_Set $\left(\right.$ seeds, $\left.X_{P I C}\right)$

7: end function

Algorithm 3: ASIA on PIC-D

4.3 Column Classification Column classification is similar to using SSL for entity classification, except that our system is tested by finding a class label for an entire column of a table - a column which contains entities of the same (unknown) type. This operation is useful in many contextse.g., in schema integration, or in indexing semi-structured web pages with relevant terms for retrieval.

Algorithm 4 describes our approach. SVM classifiers are learned as in the SSL learning case for each class of interest. Given a novel column $c=\left\{x_{1}, \ldots, x_{k}\right\}$, the PIC-D representation is retrieved for each entity $x_{i}$. Note that some entities may not correspond to nodes in the original tripartite graph; these entities are simply ignored. To classify column $c$, we find the centroid of all the $x_{i}$ 's that are represented in the PIC-D space, and classify that centroid with the SVM classifier. This is a very inexpensive operation, since SVM uses linear classifier.

In the experiments we compare this approach to performing SVM classification in the original space. In addition to classifying the centroid of the examples, we also consider taking the majority classification.

1: function Classify_Table_Column $\left(c, X_{P I C}\right.$, svm_model $): Y_{c}$

2: Input: $c$ : Query Table Column (Set of entities),

$X_{P I C}$ : Low dimensional PIC-D embedding of $E$,

svm_model: SVM classifier trained on a fraction of $X_{P I C}$

3: Output: $Y_{c}$ : Class label of $c$

4: $x_{c}=X_{P I C}($ row,$:)$, row $\in c$

5: $x_{\text {centroid }}=$ centroid $\left(x_{c}\right)$

6: $Y_{c}=\operatorname{predict}\left(\right.$ svm_model,$\left.x_{\text {centroid }}\right)$

7: end function

Algorithm 4: Column Classification using PIC-D

Table 1 shows the summary of all tasks being represented as a set of simple operations on PIC-D representation:

\section{Experiments}

In this section we evaluate the performance of the PIC-D representation on publicly available and extensively labeled datasets described in Section 5.1. Then in Section 5.2 we will evaluate whether the PIC-D representation is 


\begin{tabular}{|l|l|l|}
\hline Task & Training & Testing \\
\hline SE & PIC-D & Centroid(entity-set) + K-NN(centroid) \\
\hline ASIA & $\begin{array}{l}\text { PIC-D + } \\
\text { Index HCD }\end{array}$ & $\begin{array}{l}\text { seeds = top-k-entities( lookup concept in HCD) } \\
+ \text { SE(seeds) }\end{array}$ \\
\hline COL- & PIC-D + & \\
CLASS & Train_SVM & centroid(column) + Predict_SVM(centroid) \\
\hline
\end{tabular}

Table 1: Summary of tasks

\begin{tabular}{|l|r|r|r|r|}
\hline Dataset & $\begin{array}{r}\text { Toy } \\
\text { Apple }\end{array}$ & $\begin{array}{r}\text { Delicious_- } \\
\text { Sports }\end{array}$ & $\begin{array}{r}\text { ASIA_ } \\
\text { INT }\end{array}$ & $\begin{array}{r}\text { Clueweb_ } \\
\text { Sports }\end{array}$ \\
\hline \# HTML pages & 574 & $21 \mathrm{~K}$ & $121 \mathrm{~K}$ & $918 \mathrm{~K}$ \\
\hline$|\boldsymbol{X}|:$ \# entities & 14,996 & 438 & 14,906 & 30,382 \\
$|\boldsymbol{C}|:$ \# table-columns & 156 & 925 & 8,087 & 78,423 \\
$|(\boldsymbol{x}, \boldsymbol{c})|:$ \# $(\boldsymbol{x}, \boldsymbol{c})$ edges & 70,551 & 5,546 & 90,902 & 566,080 \\
\hline$\left|\boldsymbol{Y}_{\boldsymbol{s}}\right|:$ \# suchas concepts & 2348 & 1649 & 3,868 & 21,454 \\
$\left|\left(\boldsymbol{x}, \boldsymbol{Y}_{\boldsymbol{s}}\right)\right|:$ \# $\left(\boldsymbol{x}, \boldsymbol{Y}_{\boldsymbol{s}}\right)$ edges & 7683 & 4799 & 18,345 & 107,810 \\
\hline$\left|\boldsymbol{Y}_{\boldsymbol{n}}\right|:$ \# NELL Classes & 11 & 3 & 23 & 23 \\
$\left|\left(\boldsymbol{x}, \boldsymbol{Y}_{\boldsymbol{n}}\right)\right|:$ \# $\left(\boldsymbol{x}, \boldsymbol{Y}_{\boldsymbol{n}}\right)$ pairs & 419 & 39 & 691 & 977 \\
\hline$\left|\boldsymbol{Y}_{\boldsymbol{c}}\right|:$ \# manual column labels & 31 & 30 & - & - \\
$\left(\boldsymbol{c}, \boldsymbol{Y}_{\boldsymbol{c}}\right)$ : \# $\left(\boldsymbol{c}, \boldsymbol{Y}_{\boldsymbol{c}}\right)$ pairs & 156 & 925 & - & - \\
\hline
\end{tabular}

Table 2: Datasets Statistics

scalable in terms of pre-processing time and is it a reasonable representation of the original dataset. Next we will go through evaluation of similarity queries like Set expansion, ASIA and column classification in Sections 5.3, 5.4 and 5.5 resp. executed on the PIC-D representation.

5.1 Datasets We use the Toy_Apple, Delicious_Sports, ASIA_INT, Clueweb_Sports and Hyponym Concept datasets made publicly available by [5]. Table 2 shows the statistics of these datasets. Numbers for $\left|Y_{s}\right|$ and $\left|\left(x, Y_{s}\right)\right|$ are derived using the Hyponym Concept Dataset. Further we use an existing knowledge base NELL [3] for retrieving seed examples for a set of concepts to be used in semi-supervised learning. Statistics regarding this data are represented as $\left|Y_{n}\right|$ and $\left|\left(x, Y_{n}\right)\right|$. To do a quantitative evaluation on multiple tasks, we manually labeled the table columns from Toy_Apple and Delicious_Sports datasets, denoted by $\left|Y_{c}\right|$ and $\left|\left(c, Y_{c}\right)\right|$.

5.2 PIC-D embeddings Here we will first go through the pre-processing times for creating PIC-D embeddings. Next we will see how to tune the number of dimensions in PIC-D (parameter $m$ from the Algorithm 1 ). Finally we will evaluate whether PIC-D embeddings are reasonable based on their performance on a semi-supervised learning task.

\begin{tabular}{|l|r|r|r|r|}
\hline Dataset & Toy_ & Delicious_ \\
Apple & Sports & ASIA_ & INT & $\begin{array}{r}\text { Clueweb_ } \\
\text { Sports }\end{array}$ \\
\hline \# edges & 78,234 & 10,345 & 109,247 & 673,890 \\
$\boldsymbol{n}:$ \# total dimensions & 2,504 & 2,574 & 11,955 & 99,877 \\
$\boldsymbol{m}=\sqrt{\boldsymbol{n}}$ & 51 & 51 & 110 & 317 \\
\hline Total time to create & & & & \\
PIC-D embedding (msec) & 49.7 & 53 & 69.7630 & 365.2 \\
\hline Avg. time per & & & & \\
PIC embedding (msec) & 0.487 & 0.0520 & 0.3171 & 0.0576 \\
\hline
\end{tabular}

Table 3: Time statistics for PIC-D embeddings

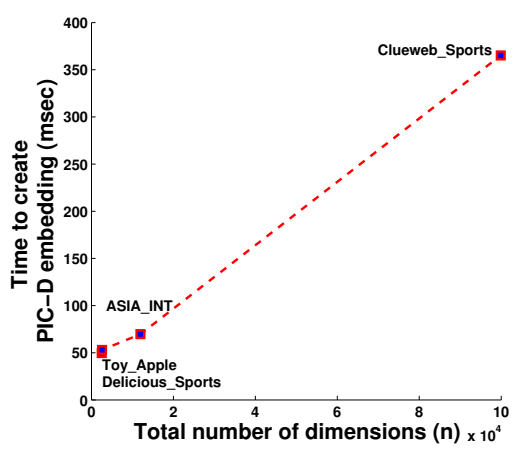

Figure 3: Time to create PIC-D for various dataset sizes

Scalability Here we evaluate the scalability of our data representation by recording the time taken to create the PIC-D embeddings of four varying sized datasets. Table 3 shows the statistics of all four datasets in terms of number of edges, number of dimensions, number of PIC-D dimensions $(m)$ and total time taken to create PIC-D embeddings. The plot in Figure 3 shows that total time required to create PIC-D embeddings grows linearly with number of dimensions of the dataset.

How many dimensions (m) in PIC-D? We use Semisupervised learning (SSL) task as a way to evaluate the the effectiveness of the PIC-D representation. Carlson et al describe NELL, a semi-supervised information extraction system for web data that works by using SSL to classify entity names found on the web [3]. We used a version of the NELL knowledge base to provide labels for entities in our datasets. To evaluate the PIC embeddings in terms of predicting these classes, we compared the performance of an SVM classifier on the PIC embeddings (named SVM+PIC-D) vs. the original high-dimensional dataset (named SVM-baseline); in SVM-baseline the hyponyms and table-columns associated with an entity are simply used as features. The number of iterations $t$ for PIC were set to $t=5$ in these experiments.

Algorithm 2 internally uses a parameter $m$ i.e. number of PIC dimensions per bipartite graph. We study the SSL performance varying number of PIC dimensions on all four datasets. Figure 4 (a) shows the results on Delicious_Sports dataset. We can see that as the number of PIC dimensions increase, performance of the classifier improves for various values of training percentage and when $m=110$ implying total number of dimensions in the PIC-D representation are 220 , the performance is very similar to SVM-Baseline.

Since SSL data is not always available, we performed some experiments to determine how quickly $m$ grows with the total number of dimensions $n$. Figure 4 (b) shows the effect of varying $m$ on all four datasets with $20 \%$ of the training data available (and the remaining $80 \%$ as test). On these datasets, when $m=\sqrt{n}$, SVM+PIC-D performs com- 


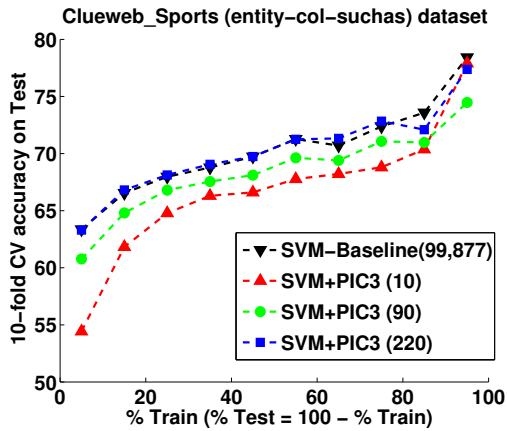

(a) Clueweb_Sports: Varying \#PIC dimensions

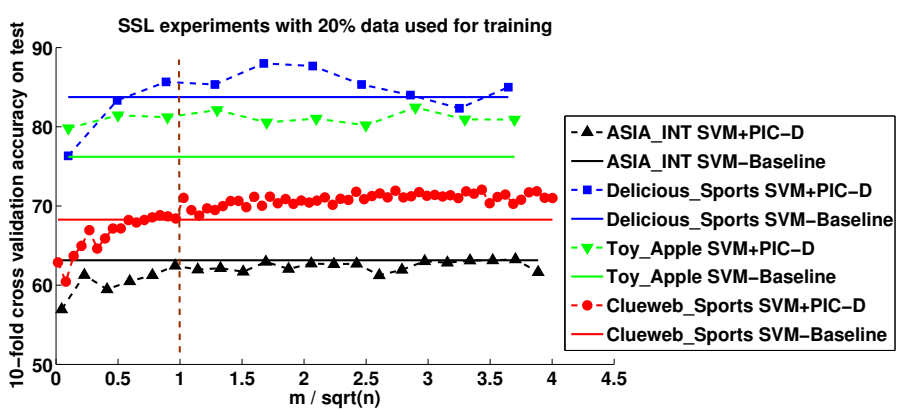

(b) Choosing \#PIC dimensions given a dataset

Figure 4: SSL Task: PIC-D embeddings of varying sizes

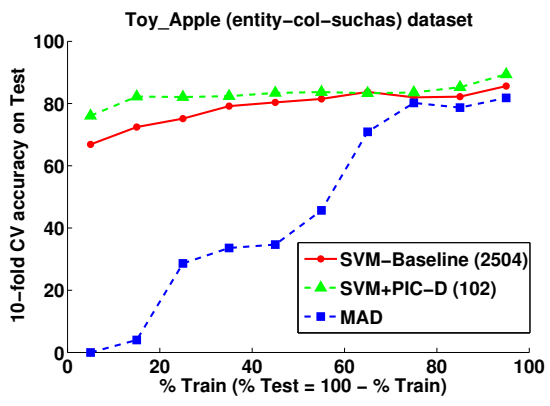

(a) Toy_Apple dataset

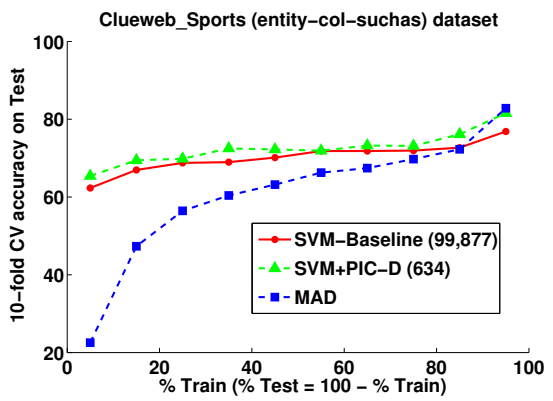

(b) Clueweb_Sports dataset

Figure 5: SSL Task: Comparison of all 3 methods

parable to or better than SVM-Baseline. In all further experiments, therefore, we set $m=\sqrt{n}$. To make sure that the embeddings are distinct from each other, we computed the rank of the PIC-D embeddings and compared it against maximum possible rank of the embeddings. We found that for embeddings of size $m=\sqrt{n}$, rank of the embedding equals the maximum possible rank, hence ensuring the distinct values in the embeddings.

Semi-Supervised Learning with tuned parameter (m) Henceforth we always pick $m=\sqrt{n}$, where $n$ is the total number of dimensions in the dataset. Now let us see with this fixed value of $m$, how effective the PIC-D representation is on the task of Semi-Supervised Learning. Figure 5 shows the plot of accuracy vs. train- ing size for Toy_Apple and Clueweb_Sports datasets. We can see that SVM+PIC-D performs better than baseline with less training data, hence is better in SSL scenarios. Both these methods perform better than MAD for small training size. We observed very similar results for ASIA_INT and Delicious_Sports datasets, SVM+PIC-D method being comparable or better than SVM-Baseline, and better than MAD (plots omitted due to space constraints).

Also note that the PIC-D embedding reduces the number of dimensions to square root of actual number of dimensions. In these experiments, we use 10 -fold cross validation for all training set sizes by randomly sampling $x \%$ of training data in each fold. Note that when we say training percentage $=x$, we sample $x \%$ of data per class label. Hence we can say that the PIC-D representation is reasonable for these datasets and it can represent the underlying class-label distribution in small number of dimensions.

5.3 Set Expansion We manually labeled every table column from Delicious_Sports and Toy_Apple datasets. These labels are referred to as $Y_{c}$ in Table 2. This also gives us labels for the entities residing in these table-columns. For this task we construct queries which contain all entities from a single table column $c$, and use label $y_{c}$ assigned to column $c$ to evaluate correctness. (All columns $c$ of Delicious_Sports and Toy_Apple datasets are manually labeled.)

One baseline approach runs K-Nearest Neighbor on the original high-dimensional dataset (referred to as K-NNBaseline). We also consider a use of MAD for this task, which is described below.

\subsubsection{Unsupervised Modified Adsorption (MAD) for Set} Expansion SEAL [19] performs set expansion by performing web queries, recognizing "wrappers" for semi-structured pages, building a graph similar to the entity-tableColumn portion of our graph, and then ranking nodes in the graph using a simple label propagation technique. Inspired by this approach, we adapted the MAD algorithm [14], a state-ofthe art semi-supervised learning method, to the set expansion 


\begin{tabular}{|l|l|r|r|r|r|}
\hline \multirow{2}{*}{ Task } & Method & \multicolumn{2}{|c|}{ Delicious_Sports } & \multicolumn{2}{|c|}{ Toy_Apple } \\
\cline { 3 - 6 } & & $\begin{array}{r}\text { Avg. Query } \\
\text { Time (msec) }\end{array}$ & $\begin{array}{r}\text { Speedup } \\
\text { of PIC-D }\end{array}$ & $\begin{array}{r}\text { Avg. Query } \\
\text { Time (msec) }\end{array}$ & $\begin{array}{r}\text { Speedup } \\
\text { of PIC-D }\end{array}$ \\
\hline \multirow{2}{*}{ Set Expansion } & K-NN+PIC-D & 12.1 & - & 72.8 & - \\
& K-NN-Baseline & 164.4 & $\mathbf{1 3 . 5}$ & 17578.3 & $\mathbf{2 4 1 . 5}$ \\
& MAD & 1902.4 & $\mathbf{1 5 7 . 2}$ & 4801.9 & $\mathbf{6 5 . 9}$ \\
\hline ASIA & K-NN+PIC-D & 20.0 & - & - & - \\
& K-NN-Baseline & 56.0 & $\mathbf{2 . 8}$ & - & - \\
& MAD & 6000.0 & $\mathbf{3 0 0 . 0}$ & - & - \\
\hline COL-CLASS & SVM+PIC-D+Centroid & 0.1 & - & 3.8 & $\mathbf{1 4 . 9 4}$ \\
& SVM-Baseline+Centroid & 1.2 & $\mathbf{1 2}$ & 56.8 & $\mathbf{1 4}$ \\
& & &
\end{tabular}

Table 4: Comparison of query times

task. Following Talukdar et al. [15], we adapt MAD for unsupervised learning by associating each table-column node with its own id as a label, and propagating these labels to other table-columns. MAD also includes a "dummy label", so after propagation every table-column $T_{q}$ will be labeled with a weighted set of table-column ids $T_{s_{1}}, \ldots T_{s_{n}}$ (including its own id), and a weight for the "dummy label".

We denote MAD's weight for associating table-column id $T_{s}$ with table column $T_{q}$ as $P\left(T_{s} \mid T_{q}\right)$, and consider the ids $T_{s_{1}}, \ldots T_{s_{k}}$ with a weight higher than the dummy label's weight. We consider $e_{1}, e_{2}, \ldots e_{n}$, the union of entities present in columns $T_{s_{1}} \ldots T_{s_{k}}$, and rank them in descending order score, where $\operatorname{score}\left(e_{i}, T_{q}\right)=\sum_{j: e_{i} \in T_{s_{j}}} P\left(T_{s_{j}} \mid T_{q}\right)$.

5.3.2 Comparison of Various Methods Table 4 presents the running time results for all three methods on 272 set expansion queries from Delicious_Sports dataset and 152 queries on Toy_Apple dataset. K-NN+PIC-D method incurs a small amount of pre-processing time $(0.053$ and $0.487 \mathrm{sec}$. $)$ to create embeddings and compared to other two methods it is very fast at the query time. The numbers show average query times for both the datasets. Note the speedup numbers for K-NN+PIC-D method, indicating the speedup of $13 x$ to 240x over K-NN-Baseline and 65x to 150x over MAD. Thus the PIC-D representation results in very fast execution of similarity queries.

Next we compare the three methods in terms of precision and recall of set expansion. Figure 6(a) shows the aggregate Precision-Recall curves for all 3 methods on the Set Expansion task on Delicious_Sports dataset. The 272 queries that we executed belong to 12 different column-labels. We first aggregate the precision values of all queries in a single class, and Figure 6(a) shows the plots of macro-averaging these precision recall curves across 12 classes. We can see that MAD algorithm performs the best in terms of precision recall curve, at the expense of drastic increase in run time of queries (Table 4). K-NN+PIC-D produce comparable results to K-NN-Baseline along with the speedup at query time. Table 5 shows some example queries and results produced by K-NN+PIC-D method on Clueweb_Sports dataset.
Seed Entities: Expanded entity set by K-NN+PIC-D method Arsenal, Liverpool, Manchester United: Middlesbrough, Man United, Blackburn Rovers, Manchester City, Tottenham, West Brom, Tottenham Hotspur, Bolton Wanderers, Newcastle United, Blackburn, Bolton, Birmingham City, Aston Villa, Chelsea Fc, Sunderland, Sheffield United, Leicester City, Everton, Chelsea, Harlequins, ...

France, UK, Denmark: Brazil, Malaysia, Indonesia, Norway, Switzerland, Great Britain, Thailand, Finland, Argentina, Belgium, Romania, Korea, Germany, Austria, Chile, Lithuania, Senegal, ...

MSN, Google, Yahoo: Qas, Mitre, Cosco, Cerberus, Cdt, Garrett, Sportingbet, Excelsior, Genzyme, Gt, Broad, Ge, Bruno, Nortel, Level 3, Nec, Foster, Renault, Ricardo, Persepolis, Coca Cola, Nike, ...

Penn State, Michigan, Princeton: Oklahoma State, Clemson, USC, Columbia, Michigan State, LSU, Dartmouth, Ohio State, Cambridge, Florida State, Wake Forest, Auburn, Vanderbilt, Duke, Hampshire, UCLA, Syracuse, Oxford, Pitt, North Carolina State, Mississippi State, ...

San Francisco, Seattle, Houston: Detroit, Philadelphia, Denver, Boston, Atlanta, Nashville, Minneapolis, New Orleans, San Diego, Dallas, Miami, Pittsburgh, Cincinnati, Los Angeles, Chicago, Oakland, Tampa, Cleveland, Portland, Tokyo, Des Moines, Kansas City, Charlotte, ...

\section{Table 5: Set Expansion on Clueweb_Sports Dataset}

5.4 Automatic Set Instance Acquisition For the automatic set instance acquisition (ASIA) task, we use conceptnames from Hyponym Concept Dataset $\left(Y_{s}\right)$ as queries. Similar to the Set Expansion task, we compare K-NN+PIC-D to the K-NN-Baseline and MAD methods.

5.4.1 MAD used for ASIA task To use MAD for this task, the concept name $Y_{s}$ is injected as label for the ten entities that co-occur most with $Y_{s}$, and the label propagation algorithm is run. Each entity $e_{i}$ that scores higher than the dummy label is then ranked based on the probability of the label $Y_{s}$ for that entity.

Table 4 shows that K-NN+PIC-D takes on average only $20 \mathrm{msec}$. to run a ASIA query as compared to $56 \mathrm{msec}$. by K-NN-Baseline and $6000 \mathrm{msec}$. by MAD. These query times are averaged over a set of 25 ASIA queries from Delicious_Sports dataset. Figure 6 (b) shows the comparison of all three methods on the ASIA task in terms of macro-averaged precision recall curves (following the same procedure for averaging as in set expansion). K$\mathrm{NN}+\mathrm{PIC}-\mathrm{D}$ generally outperforms K-NN-Baseline. MAD 


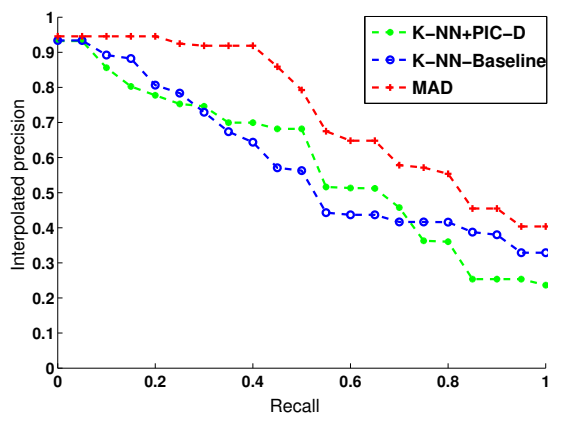

(a) Task: Set Expansion

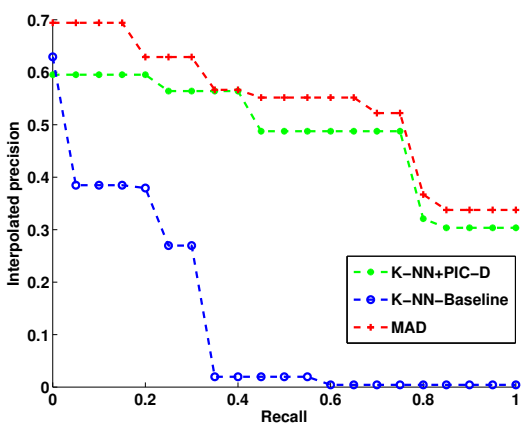

(b) Task: ASIA

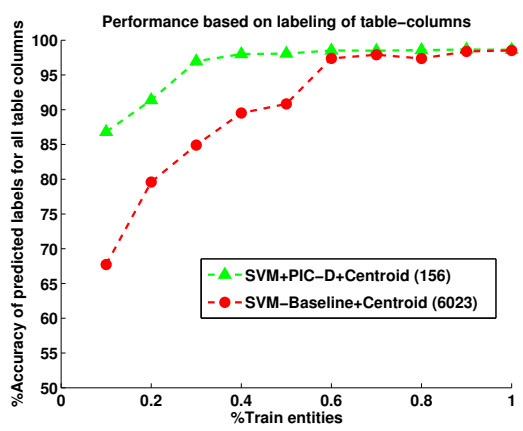

(c) Task: COL-CLASS

Figure 6: Results on Delicious_Sports dataset

\begin{tabular}{|c|c|c|}
\hline $\begin{array}{l}\text { Concept } \\
\text { Name }\end{array}$ & $\begin{array}{l}\text { Seed Enti- } \\
\text { ties }\end{array}$ & K-NN + PIC-D: Expanded entity set \\
\hline Sports & $\begin{array}{l}\text { Football, } \\
\text { Basketball, } \\
\text { Soccer }\end{array}$ & $\begin{array}{l}\text { Softball, Ice Hockey, Volleyball, Skating, } \\
\text { Martial Arts, Windsurfing, Hunting, Strength } \\
\text { Sports, Lacrosse, Dodgeball, Curling, ... }\end{array}$ \\
\hline City & $\begin{array}{l}\text { New York, } \\
\text { London, } \\
\text { Los Angeles }\end{array}$ & $\begin{array}{l}\text { Tokyo, Grand Rapids, San Jose, Memphis, } \\
\text { Long Beach, Ft Lauderdale, Southern New } \\
\text { England, Minnesota, Washington, ... }\end{array}$ \\
\hline Country & $\begin{array}{l}\text { United } \\
\text { States, } \\
\text { Canada, } \\
\text { India }\end{array}$ & $\begin{array}{l}\text { Australia, Dr Congo, Argentina, Colombia } \\
\text { North Korea, China, Malaysia, Pakistan, Nor- } \\
\text { way, Philippines, Iceland, Egypt, Ecuador, In- } \\
\text { donesia, Vietnam, South Africa, Brasil, ... }\end{array}$ \\
\hline $\begin{array}{l}\text { Outdoor } \\
\text { Recreation }\end{array}$ & $\begin{array}{l}\text { Hunting, } \\
\text { Fishing, } \\
\text { Skiing }\end{array}$ & $\begin{array}{l}\text { Cross Country, Martial Arts, Ice Hockey, Cro- } \\
\text { quet, Curling, Climbing, Lacrosse, Softball, } \\
\text { Basketball, Golf, Windsurfing, Baseball, ... }\end{array}$ \\
\hline $\begin{array}{l}\text { Major } \\
\text { European } \\
\text { Countries }\end{array}$ & $\begin{array}{l}\text { France, Ger- } \\
\text { many, UK }\end{array}$ & $\begin{array}{l}\text { Slovakia, Thailand, Israel, Czech Republic, } \\
\text { United States, Brazil, Iceland, Belgium, Hong } \\
\text { Kong, Canada, Serbia, Uruguay, ... }\end{array}$ \\
\hline Leagues & $\begin{array}{l}\text { NFL, } \\
\text { Premier } \\
\text { League, } \\
\text { NBA }\end{array}$ & $\begin{array}{l}\text { NHL, NASCAR, NHRA, NCCA, PGA, Sports } \\
\text { Illustrated, .... }\end{array}$ \\
\hline
\end{tabular}

Table 6: ASIA task on Clueweb_Sports dataset

is slightly batter than K-NN+PIC-D at the expense of much longer query times. These results show that $\mathrm{K}$ $\mathrm{NN}+\mathrm{PIC}-\mathrm{D}$ achieves comparable quality results w.r.t MAD by reducing the query time by a factor of 300 (Table 4). Table 6 shows some example ASIA queries and output produced by K-NN+PIC-D method on Clueweb_Sports dataset.

5.5 Column Classification For this task we use the manual labels $\left(Y_{c}\right)$ assigned to all table columns of Delicious_Sports and Toy_Apple datasets. These labels are further extended to entities appearing in those table columns. Column Classification (COL-CLASS) task is to predict $Y_{c}$ for a given table column $c$, using a classifier trained on few example entities for each $Y_{c}$.

We refer to our method described in Algorithm 4 as SVM+PIC-D+Centroid. Similar to SSL task, we create a version of this method which trains SVM classifier and predicts class label of the column in original data space. This method is referred to as SVMBaseline+Centroid. Figure 6 (c) shows the performance of all these two methods on Delicious_Sports dataset. It can be seen that SVM+PIC-D+Centroid gives a comparable or better performance when compared to SVMBaseline+Centroid. Note that along with the superior quality results, SVM+PIC-D+Centroid achieves a query-time speedup of $12 \mathrm{x}$ to $14 \mathrm{x}$ as shown in Table 4

We also tried a variant of both these methods where prediction of the label of a table column is the majority of labels predicted for each of its entities. These methods are referred to as SVM+PIC-D+Majority and SVM-Baseline+Majority resp. We observed in our experiments that there is no significant difference between SVM+PIC-D+Centroid and SVM+PIC-D+Majority, while SVM-Baseline+Majority performs slightly better than SVMBaseline+Centroid. The performance of these methods on Toy_Apple dataset followed very similar trends (plots omitted due to space constraints). Overall, PIC-D based methods performed better than baseline methods for both the datasets in terms of query-time and prediction accuracy.

\section{Related Work}

This work uses the Power Iteration Clustering (PIC) algorithm by Lin and Cohen [11]. We propose a low-dimensional representation for entities based on the embedding used internally by the PIC algorithm. Briefly, PIC assigns nearby values to similar nodes, thus producing a one-dimensional embedding of a graph. Clustering is then performed in this one-dimensional space. Extension of PIC for bipartite graphs is demonstrated by Lin and Cohen [12].

PIC is very scalable, and in past experiments it has shown to be comparable to spectral clustering methods for certain network datasets, and superior to traditional $k$-means clustering on certain text-clustering tasks (where a text corpus is represented as a bipartite graph with nodes that are terms or documents.) It has also been previously shown that the performance of PIC can be improved by using multiple random starting points, thus producing a low-dimensional (but not one-dimensional) embedding of a graph [1]. This paper considers D-partite graphs, and evaluates new oper- 
ations (other than clustering) on the embedded space. We note that many of the tasks considered here are quite different from cases where PIC was previously used, in that there are many small clusters rather than a few large ones. We also showed that similarity queries can be executed extremely fast on the PIC-D embedding.

Another line of related work concerns information extraction from semi-structured web data sources. WebTables [2] demonstrated the utility of large corpora of HTML tables, and such data has been used for various inference tasks by several other researchers [19, 9, 6, 16], including several of the individual tasks discussed above, notably set expansion using semi-structured data [19]. Set expansion has been used for tasks such as answering entity-list completion queries [4], and the semi-structured data used in set expansion has been extended with other sources, such as web search logs [7]. Wang and Cohen also used semi-structured data in an extended set-expansion system, in conjunction with hyponym data collected at query time, for the automatic set instance acquisition task [18].

The combination of tables and hyponym data was also used by [14] to learn class-instance facts of the form "entity $x$ is a member of concept $c$ ", using a label propagation method called Modified Adsorption (MAD). Here we use the same representation as one of our baselines, and consider using MAD on this graph to propagate labels, but consider a broader set of tasks, and compare to other representations as well. WebSets [5] also demonstrated unsupervised classinstance fact extraction using HTML tables and Hearst patterns [8], and the data we use for evaluation is taken from the WebSets project [5]. Here again we consider using similar underlying data, but consider a different set of tasks.

\section{Conclusions}

In this paper, we propose a single low-dimensional representation for entities found in semi-structured data on the web. Further we show that a single, efficiently-constructible representation, the PIC-D representation, can be used for answering similarity queries like set expansion, automatic set instance acquisition, and column classification.

We present experiments on large collections of tables and hyponym data published by [5]. Our proposed approach gives comparable precision values with respect to the task specific baselines. Additionally, it gives up to two orders of magnitude improvement in query response time. The experimental success of our approach is especially encouraging given that there are very few parameters to tune in building the representation (only the number of iterations $t$ for PIC and the number of PIC dimensions $m$, set to $t=5$ and $m=\sqrt{n}$ in the experiments).

These initial results raise a number of interesting questions. A plausible research direction would be to use the PIC-D representation with many more "views" of entity data (for instance, features describing the content of an entity name, or properties derived from large broadcoverage knowledge bases such as FreeBase). One could also investigate class-instance fact acquisition using the PIC-D representation.

Acknowledgments This work is supported in part by the Intelligence Advanced Research Projects Activity (IARPA) via Air Force Research Laboratory (AFRL) contract number FA8650-10-C-7058. The U.S. Government is authorized to reproduce and distribute reprints for Governmental purposes notwithstanding any copyright annotation thereon. This work is also partially supported by the Google Research Grant. The views and conclusions contained herein are those of the authors and should not be interpreted as necessarily representing the official policies or endorsements, either expressed or implied, of Google, IARPA, AFRL, or the U.S. Government.

\section{References}

[1] R. Balasubramanyan, F. Lin, and W. W. Cohen. Node clustering in graphs: An empirical study. Workshop on Networks Across Disciplines in Theory and Applications, NIPS, 2010

[2] M. J. Cafarella, E. Wu, A. Halevy, Y. Zhang, and D. Z. Wang. Webtables: Exploring the power of tables on the web. PVLDB, 2008.

[3] A. Carlson, J. Betteridge, R. C. Wang, E. R. Hruschka, Jr., and T. M. Mitchell Coupled semi-supervised learning for information extraction. In WSDM, 2010.

[4] B. Dalvi, J. Callan, and W. Cohen. Entity list completion using set expansion techniques. In TREC, 2010.

[5] B. Dalvi, W. Cohen, and J. Callan. Websets: Extracting sets of entities from the web using unsupervised information extraction. In WSDM, 2012.

[6] R. Gupta and S. Sarawagi. Joint training for open-domain extraction on the web: exploiting overlap when supervision is limited. In WSDM, 2011.

[7] Y. He and D. Xin. Seisa: set expansion by iterative similarity aggregation. WWW, 2011.

[8] M. A. Hearst. Automatic acquisition of hyponyms from large text corpora. In $A C L, 1992$.

[9] G. Limaye, S. Sarawagi, and S. Chakrabarti. Annotating and searching web tables using entities, types and relationships. PVLDB, 2010.

[10] F. Lin. Scalable methods for graph-based unsupervised and semi-supervised learning. In PhD thesis, 2012. http://www.lti.cs.cmu.edu/ research/thesis/2012/frank_lin.pdf

[11] F. Lin and W. W. Cohen. Power iteration clustering. ICML, 2010.

[12] F. Lin and W. W. Cohen. A very fast method for clustering big text datasets. ECAI, 2010

[13] N. Otsu. A threshold selection method from gray-level histograms. In IEEE Transactions on Systems, Man and Cybernetics, 1979.

[14] P. Talukdar and K. Crammer. New regularized algorithms for transductive learning. In ECML-PKDD. 2009.

[15] P. P. Talukdar, Z. G. Ives, and F. Pereira. Automatically incorporating new sources in keyword search-based data integration. SIGMOD, 2010.

[16] P. Venetis, A. Halevy, J. Madhavan, M. Paşca, W. Shen, F. Wu, G. Miao, and C. Wu. Recovering semantics of tables on the web. $V L D B, 2011$.

[17] R. C. Wang and W. W. Cohen. Language-independent set expansion of named entities using the web. In ICDM, 2007.

[18] R. C. Wang and W. W. Cohen. Automatic set instance extraction using the web. In $A C L / A F N L P, 2009$.

[19] R. C. Wang and W. W. Cohen. Character-level analysis of semi-structured documents for set expansion. In EMNLP, 2009. 